recommended in a child with varicella who presents with reddening of an eye or blurred vision, to exclude papillitis or necrotizing retinitis. Prompt antiviral therapy may help to preserve some residual vision and prevent extension of the lesion. Systemic corticosteroids are not generally advocated, and possible benefits of a brief course used in the above child with a fulminant retinopathy would require confirmation by controlled trial.

\title{
HERPES SIMPLEX VIRUS REACTIVATION AFTER HEMISPHERECTOMY FOR INTRACTABLE SEIZURES
}

Researchers at the Cleveland Clinic report a 23-month-old immunocompetent boy with reactivated herpes simplex virus encephalitis (HSE) after a subtotal hemispherectomy for seizures related to HSE-associated right middle cerebral artery infarction at $\bar{i}$ months of age. The diagnosis had been confirmed by HSV PCR from CSF, and he had completed a 21 day couse of iv acyclovir. Acyclovir and dexamethasone were administered routinely at time of surgery. Fever developed on postoperative day 1 and persisted despite continued acyclovir. CSF HSV PCR was positive for type 1 on postop day 8 and negative day 11 . At 3 weeks he was doing well with no seizures and was afebrile. Resected tissue showed multiple infarctions and microglial nodules, consistent with HSE history. (Gong T, Bingaman W, Danziger-Isakov L, Tuxhorn I, Goldfarb J. Herpes simplex virus reactivation after subtotal hemispherectomy in a pediatric patient. Pediatr Inf Dis Jrnl Dec 2010;29(12):1148-1150). (Respond:Johanna Goldfarb MD, 9500 Euclid Ave, S25, Cleveland, OH 44195. E-mail: goldfaj@ccf.org).

COMMENT. Reactivation of HSV after HSE is unusual and typically occurs immediately after treatment of a primary encephalitis. Cases with shorter courses of acyclovir may be susceptible. The authors cite one previous case in the literature of HSV reactivation after epilepsy surgery for mesial temporal sclerosis. The primary infection occurred at 16 months of age and reactivation 6 years later after hippocampectomy and gyrus corticotomy, the previous site of herpes infcction. (Bourgeois $\mathrm{M}$ et al. Neurosurgery 1999;44:633-635).

HSV reactivation should be ruled out by repeated CSF PCR in patients with persistent fever and irritability following epilepsy surgery. Transient immunosuppression complicating perioperative steroid therapy is suggested as a mechanism for HSV reactivation following hemispherectomy.

\section{DEMYELINATING DISORDERS}

\section{COGNITIVE IMPAIRMENT IN MULTIPLE SCLEROSIS AND MRI}

The utility of MRI techniques to monitor cognitive impairment progression in MS over time and to assess treatment is reviewed by researchers at University Hospital, San Raffaele, Milan, Italy; State University of New York, Buffalo; University of New Jersey, Newark; Leiden Institute for Brain and Cognition, the Netherlands; and University College and Institute of Neurology, Queen Square, London, UK. Focal white matter 